\title{
Bridging prediction and attention in current research on perception and action
}

Citation for published version (APA):

Schröger, E., Kotz, S. A., \& SanMiguel, I. (2015). Bridging prediction and attention in current research on perception and action. Brain Research, 1626, 1-13. https://doi.org/10.1016/j.brainres.2015.08.037

Document status and date:

Published: 01/01/2015

DOI:

10.1016/j.brainres.2015.08.037

Document Version:

Publisher's PDF, also known as Version of record

Document license:

Taverne

Please check the document version of this publication:

- A submitted manuscript is the version of the article upon submission and before peer-review. There can be important differences between the submitted version and the official published version of record.

People interested in the research are advised to contact the author for the final version of the publication, or visit the DOI to the publisher's website.

- The final author version and the galley proof are versions of the publication after peer review.

- The final published version features the final layout of the paper including the volume, issue and page numbers.

Link to publication

\footnotetext{
General rights rights.

- You may freely distribute the URL identifying the publication in the public portal. please follow below link for the End User Agreement:

www.umlib.nl/taverne-license

Take down policy

If you believe that this document breaches copyright please contact us at:

repository@maastrichtuniversity.nl

providing details and we will investigate your claim.
}

Copyright and moral rights for the publications made accessible in the public portal are retained by the authors and/or other copyright owners and it is a condition of accessing publications that users recognise and abide by the legal requirements associated with these

- Users may download and print one copy of any publication from the public portal for the purpose of private study or research.

- You may not further distribute the material or use it for any profit-making activity or commercial gain

If the publication is distributed under the terms of Article $25 \mathrm{fa}$ of the Dutch Copyright Act, indicated by the "Taverne" license above, 


\section{Editorial}

\section{Bridging prediction and attention in current research on perception and action}

\section{A R T I C L E I N F O}

Available online 5 September 2015

\begin{abstract}
A B S T R A C T
Prediction and attention are fundamental brain functions in the service of perception and action. Theories on prediction relate to neural (mental) models inferring about (present or future) sensory or action-related information, whereas theories of attention are about the control of information flow underlying perception and action. Both concepts are related and not always clearly distinguishable. The special issue includes current research on prediction and attention in various subfields of perception and action. It especially considers interactions between predictive and attentive processes, which constitute a newly emerging and highly interesting field of research. As outlined in this editorial, the contributions in this special issue allow specifying as well as bridging concepts on prediction and attention. The joint consideration of prediction and attention also reveals common functional principles of perception and action.
\end{abstract}

(c) 2015 Elsevier B.V. All rights reserved.

\section{Introduction}

Predictive and attentive processing serves to optimize perception and action. The respective theoretical constructs, prediction and attention, have a long tradition in neuroscience. The physiologist Hermann von Helmholtz emphasized the importance of unconscious inferences on information processing already in the 19th century (von Helmholtz, 1867) and the psychologist William James elaborated on the beneficial role of attention (James, 1890). Although attention and prediction lack an unambiguous definition, they can still be separated from each other. On the one hand, attention research is concerned with how large amounts of information, available to the brain, are selected and controlled to enable or aid perception and action; for example, in order to hear a specific speaker in a noisy environment or to grasp a particular object from a range of objects, task-relevant information has to be selected and prioritized relative to task-irrelevant information. One important distinction is that attention can be controlled voluntarily (e.g., "I want to listen to the sounds from the left") or that it can be captured involuntarily under particular circumstances (e.g., "My eyes jump to a suddenly emerging object in the visual field"). On the other hand, prediction research emphasizes the anticipatory nature of the information processing system and relies on the assumption that perception and action are guided by internal models that generate predictions. For example, in order to catch a flying ball, one has to anticipate the trajectory of the ball and to prepare appropriate movements that need to be executed at the correct point in time; thus, perception and action rely on predictions in order to accomplish this task. However, the assumption is that predictive processes are an intrinsic feature of the brain and come into play for any kind of perception and action as defined in the active inference theory (Friston et al., 2010).

This special issue includes current state of the art research on prediction and attention that utilized different methodological and theoretical approaches. Some of the contributions are solely devoted to either prediction or attention, while others focus on the interaction of predictive and attentive processes engaged in perception and action. The scientific contributions to this special issue are also a showcase for the diverse conceptualizations of prediction and attention in different research traditions and the diversity of experimental manipulations, which are employed to tap into these two processes. This variety is a testimony to the importance of diverse concepts in attention and prediction research, which 
have been prevalent topics in psychology, psychophysiology, and neuroscience over the last century. However, exactly this variety of methodological approaches and concepts also highlights the need to find better agreement and common ground definitions that will ultimately facilitate the integration of these different research traditions. By bringing together studies from these different traditions in this special issue, we hope to start building a bridge for similar concepts and establish the ground for a more integrative understanding of how attention and prediction may interface.

Traditionally, attention studies have investigated the processing differences between attended and unattended stimuli (for a review cf. e.g., Näätänen et al., 2002). Attention is typically directed towards a to-be-selected feature via instructions or cues, either in a sustained or trial-by-trial manner. When responses are compared between to-beselected or cued (attended) and to-be-ignored (unattended) stimuli, processing advantages are observed for the attended stimuli. Behaviorally, faster and more accurate responses are obtained, and even perceptual sensitivity can be improved. At a neurophysiological level, both increased sensory responses as well as additional, superimposed neural activity is elicited by attended stimuli. The selective enhancement for attended stimuli is often accompanied by selective inhibition of the tobe-ignored stimuli, when compared to neutral controls. Attention studies have not only been concerned with effects on stimulus-evoked responses, but have also investigated the neural activity related to attention processes themselves by analyzing brain activity in sustained attention tasks in the absence of stimuli, or in short expectation periods prior to the presentation of stimuli. In these situations, attention-related neural activations are also observed. The combined insight yielded by these studies has led to a relatively established view of attention, in which somewhat abstract representations of relevant or to-be-attended features or objects are formulated and held in association brain areas. These areas, including frontal and parietal cortices, influence the processing in lower sensory areas, modulating sensory responses positively when objects are attended.

This view of attention as a top-down mechanism is, however, closely linked to the concept of voluntary or selective attention (e.g. Näätänen, 1990). As stated above, attention can also be captured externally by particular events, which is referred to as involuntary attention. Stimuli that capture attention involuntarily lead to similar benefits as when attention is directed in a controlled manner. It is known that similar attentional processes come into play in this case, although somewhat different brain networks seem to be involved in voluntary and involuntary attention. To study the processes related to involuntary attention, unexpected or highly salient stimuli are most effectively capturing attention, and hence are typically used. In this respect, attention and prediction research has been somewhat conflated early on, given that prediction research is engaged in studying the processing differences between predicted (i.e., "expected") and unpredicted (i.e., "unexpected") stimuli.

Prediction has been primarily studied in oddball paradigms, in which a stimulation rule is established via repetitions, the most simple rule being the exact repetition of the same stimulus. Once established, stimuli violating the rule are interspersed, and, in the most simple case, replace the repeated (i.e. "standard") stimulus with a different (i.e., "deviant") stimulus. Sensory responses to deviant stimuli are enhanced compared to standard stimuli, and a variety of additional neural responses are specifically elicited by deviant stimuli, depending on the particular paradigm that is used. Shorter latency responses such as the mismatch negativity (MMN; for reviews, see e.g., Näätänen et al., 2011; Kujala et al., 2007) are interpreted as indicators of a detected regularity violation, and thus indirectly also as an indicator of the extraction of rules and operations underlying internal predictive models in the service of sensory processing and perception (e.g., Winkler, 2007). Some longer latency responses elicited by a deviant stimulus such as the P3 response (Friedman et al., 2001; Polich, 2007), have been directly related to involuntary orienting mechanisms, reflecting attention capture by the unexpected stimulus, once more exemplifying the interrelatedness of attentive and predictive processes (e.g., Escera et al., 2000).

Significant research has investigated the processes triggered by a deviant stimulus and related them to predictive processing and the violation of predictions stemming from internal predictive models (e.g., Bendixen et al., 2012). More recently, however, there has also been an increasing interest in the processing of stimuli, which are not violating but matching predictions stemming for internal predictive models (e.g., Hughes et al., 2013). This has been investigated by looking at responses elicited by the standard stimuli in oddball-type studies, but also in studies employing other types of cues that provide predictive information, e.g. using a visual warning cue to predict an upcoming sound, or investigating responses to sensory stimuli produced by one's own motor acts, e.g., pressing a button to produce a sound. A common finding in these studies is that when a stimulus is predictable, the sensory response is attenuated, which is not the case when the same stimulus is unpredictable. It has been proposed that the response attenuation of a predictable stimulus is the result of canceling any responses matching the predictions of an internal forward model, a process which is also referred to as "explaining away", as the activity elicited by this type of stimulation is explained (i.e., is predictable or already known) by the internal model and consequently canceled and not further considered for processing.

In recent years, prediction research has experienced a drastic transformation with an upsurge of interest in the idea of active inference as an account of perception. In this view, perception is the product of an interaction between the sensory input and an internal neural model of the world that generates predictions about the sensory input. This model infers causes of the sensory input, that is, representations or beliefs of an event in the outer world that has generated sensory input. The inference is based on estimates of likelihood and prior probability (prior beliefs based on our experience), which constrain and bias our interpretation of the sensory information (posterior probability). The inferred cause is used to formulate predictions about the sensory input in 
order to verify the validity of the inference. This prediction is, in turn, compared with sensory input, and potential differences between the predicted and actual input are expressed as prediction error. This error is used to update, maintain, and optimize the model (Rao and Ballard, 1999). Thus, perception is regarded as the result of a hierarchical Bayesian inference, in which bottom-up sensory input and top-down predictions are integrated in recurrent feedforward/feedback loops across different levels of the cortical hierarchy (Lee and Mumford, 2003). Analog predictive models have been formulated for motor behavior (Adams et al., 2013) and action (Friston et al., 2010), and suggested an approach towards a unified science of mind and action (Clark, 2013).

The findings collected from oddball and other prediction studies summarized above that show increased responses to unexpected and attenuated responses for expected stimuli are mostly consistent with this account of perception and, in particular, with predictive coding models, which constitute a specific instantiation of active inference (Friston, 2005). In predictive coding, the sensory signals moving along the cortical hierarchy in a feedforward manner, only represent the prediction error, that is, the difference between the predictions of the internal model, and the sensory input. Therefore, a mismatched stimulation (e.g. deviants) should elicit large prediction error responses, while responses to prediction-matching stimulation should be attenuated (i.e., explained away by the predictions). Attention also has a place in predictive coding models, where it is regarded as a mechanism weighting the gain of the prediction error signals by its precision (e.g., Feldman and Friston, 2010; Hohwy, 2013). Thus, attention represents the confidence placed in the sensory evidence, and modulates the magnitude of neural responses, increasing the gain of responses to attended stimuli, which afford high confidence.

Whichever perspective or approach, it seems clear that attention and prediction processes may have a close relation. However, a potential interaction of attention and prediction can only be properly addressed once the concepts of attention and prediction themselves are clearly defined. A great deal of confusion regarding the definition of these two concepts results from varying terminological descriptions. For example, attention is sometimes manipulated by using probabilistic information (i.e., an attentional cue informs about the likelihood of targets to appear) and it is required that participants also respond to unattended (uncued or invalid cues) target stimuli. In other words, what is regarded as a prediction manipulation in some studies, has been understood as an attention manipulation in others. Recently, some effort has been put into establishing orthogonal and specific definitions of attention and prediction that proposes to define prediction as being related to probability and attention as being related to instructional (and possibly even motivational, emotional) relevance (Summerfield and Egner 2009; Kok et al., 2012; Schröger et al., 2015). However, research on a possible interaction of attention and prediction is only beginning. The ultimate aim of this special issue is therefore to inform the interested reader about new results and developments in the field and to stimulate research that jointly considers the important concepts of prediction and attention in order to better understand how perception and action are instantiated in the human brain. With this spirit in mind, the following sections will provide a brief overview of the concepts and ideas touched upon by the work contributing to this special issue with an angle at bridging the different traditions in the respective research on prediction and attention and the current studies.

\section{Prediction}

The term prediction has been "en vogue" for a number of years now, but reference to analogous concepts has had a long tradition in cognitive psychology, psychophysiology, and the neurosciences. Diverse fields of study have alluded to predictive processing mechanisms to explain different phenomena and yet others that have not traditionally been viewed from the prism of prediction, are now being reinterpreted from this viewpoint. One example, in which prediction-related concepts have been implicated early on in human electrophysiology studies is the oddball paradigm. The discovery of the mismatch negativity (MMN), an electrophysiological response elicited by stimuli that deviate from the preceding context, exposed the existence of internal models keeping track of the regularities in sensory input and have demonstrated that deviance detection takes place preattentively. Since then, MMN theories have evolved from memory-based explanations, in which every incoming stimulus is compared to a memory trace of the preceding input (Näätänen, 1992), to explanations emphasizing the predictive nature of sensory processing, and proposing that the MMN is elicited by prediction violations based on internal models that capture sensory regularities (Winkler, 2007).

Another early example of predictive processes can be found in physiological studies of action-perception interactions investigating how sensory processing can compensate the distortions caused by an organism's own movement such as the displacement of visual objects on the retina caused by bodily or eye movements. The reafference principle (von Holst and Horst, 1950) states that an internal model predicts the sensory consequences of a planned action and uses this prediction as a sort of negative image to cancel out the respective sensory input, compensating the unwanted sensory effects of movement. Internal models predicting the sensory consequences of motor behavior have later also been given a prominent position in computational models of motor control (Miall and Wolpert, 1996). In such models, the motor commands are selected through a comparison between the predicted external consequences of the execution of the command, and the current motor goals. Prediction can also be related to learning, the most simple form of prediction being the one that can be made after learning a simple association. Indeed, studies that aim to investigate predictive processes often do so by establishing simple associations between stimuli within the course of an experiment, i.e., a particular cue is repeatedly paired with a particular stimulus. Subsequently, the processing differences between the predicted (i.e, cued) stimulus and an unpredicted (i.e., uncued or invalidly cued) stimulus are studied. Thus the term prediction is employed in this situation to refer to 
processes that have previously been viewed from the perspective of associative learning, or to phenomena that can even be related to priming.

This short overview demonstrates a simple point. The increased frequency with which the term prediction is used in different research contexts may be a good sign, insofar as it is a desirable goal to uncover general principles of brain function and the idea of the brain as a prediction machine may be one such integrative constructs with very high explanatory power. However, the indiscriminate use of the term prediction as a new "trend" also has the danger of generating confusion regarding the definition of the concept. If everything is now termed prediction, then what exactly is prediction? In this special issue we present a range of contributions addressing the involvement of predictive processes in perception and action. In this respect, we conceive prediction as a mechanism that is an integral part of sensory processing and action control. Predicting is thus a general operation, which enables or aids perception and action. By defining prediction, it is important to stress its prospective nature. To predict is to anticipate the future based on the past. This requires the existence of an internal model, which captures learned associations and regularities upon which predictions can be formed. The model can be more or less elaborated, with a one-to-one association of A preceding $\mathrm{B}$ being the simplest possible model, which would allow to predict B whenever A occurs.

Keeping this in mind, a first question to be addressed is whether the cognitive system is indeed predictive at all, and if so, how generalizable is the concept of prediction. The papers comprised in the current special issue highlight the pervasive influence of predictive processing in brain function, providing examples for its involvement in audition (e.g. Cornella et al., 2015; Bendixen et al., 2015) and vision (e.g. Kimura and Takeda, 2015; Sulykos et al., 2015) and also in motor processes (Stekelenburg and Vroomen, 2015; Van der Steen et al., 2015) and higher-order functions such as language (Huettig, 2015), showing that predictive processing is a relatively generalizable modus operandi in sensory processing across modalities and in motor control. Given this ubiquity of predictive processes, Háden et al. (2015) wonder whether forming predictions is a fundamental characteristic of how the brain learns regularities, asking whether it is an innate capacity of the human brain. To answer this question, they tested human newborns in an MMN paradigm specifically designed to leave out non-prediction based alternative interpretations. Their results indicate that regularities for sound sequences are extracted throughout the sequence by the newborn brain and predictions are formed regarding the continuation of the sequence. They conclude that predictive processing is a basic principle of perception, and an innate capacity of the human brain.

The MMN is an indicator for prediction that is located at a sensory level in a time range of around 150 (auditory) to 250 (visual) milliseconds, close to the establishment of the respective percept (Näätänen and Winkler, 1999). However, recent studies have provided evidence that predictive processing already takes place at even earlier stages of sensory processing, complementing MMN findings with measures tapping onto mid-latency responses (MLRs) of the event- related potential (ERP), which reflect activity in the primary cortex and thalamo-cortical loops. Specifically, for simple rules it has been reported that prediction effects occur already around $30 \mathrm{~ms}$ after the onset of a prediction violation (Grimm and Escera, 2012). In this special issue, Cornella et al. (2015) looked for early electrophysiological signatures of prediction for more complex regularities, and report that a complex spatial auditory regularity modulates the $\mathrm{Na}$ component of the middle latency response at around $34 \mathrm{~ms}$.

Thus there seems to be sufficient evidence that prediction is a basic principle in sensory processing, being present in different modalities, at different levels of the processing hierarchy including the very early ones, and being present already at birth as an innate capability of brain function. Nevertheless, it is still unclear how specifically prediction works, and thus a healthy debate around the somewhat undefined concepts of prediction remains. Two main types of questions can be addressed regarding prediction, namely those related to how the internal models are formed, and those related to how they are applied, that is, how the predictions stemming from the internal models affect sensory and motor processes.

A useful approach to understand how internal models are formed is to explore not only mismatch but also match effects, that is, to examine the processing of stimuli conforming to the stimulation rules. In general, the repeated presentation of a stimulus results in reduced neural responses, due to adaptation of the underlying neural populations. That is, after a stimulus is presented, the units that have just fired in response to it find themselves in a refractory state when the same stimulus is repeated, and thus responses to the subsequent presentations of the same stimulus are suppressed. This effect is also known as repetition suppression (Grill-Spector et al., 2006). There is a longstanding debate regarding whether deviance responses like the MMN truly reflect predictive processing, or they can rather be fully explained by neural adaptation effects (May and Tiitinen, 2010). Whether adaptation based or not, repetition effects may nevertheless be related to the encoding of regularities. Using an adaptation of the oddball paradigm, Haenschel et al. (2005) described an electrophysiological response, which they named repetition positivity, that increases with the number of repetitions of the standard stimuli. Such behavior is consistent with the possibility that it reflects the registration of the regularity, which would be strengthened with every subsequent repetition. However, when the rule consists on the simple repetition of the same stimulus, the effects may also be a reflection of increasing adaptation solely. In the present issue, Kimura and Takeda (2015) investigate the encoding of regularities in the visual modality, using not only a simple repetition condition, but also a more complex regularity condition in which the standard stimulus is expected to change rather than repeat in every trial. Compared to a random control condition in which no regularity was present, standards in the regular sequences elicited a reduced P2 component of the ERP, reflecting the encoding of the regularity. Additionally, they also measured responses to deviants, and reported a high correlation between match and mismatch effects, supporting 
the idea that these two effects depend on the same underlying predictive model.

The relationship between the effects observed for simple stimulus repetitions and those observed for the encoding of more complex regularities is nevertheless unclear. Viewed as a by-product of neural refractoriness, repetition suppression was considered to be a purely bottom-up phenomenon. However, recent studies have shown that it may at least partly depend on top-down expectation, that is, it is tied to predictive mechanisms (Summerfield et al., 2008). Presently, the neural mechanisms engaged to register regularities and form internal predictive models are not yet known, but it is quite possible that similar or the same neural adaptation mechanisms involved in repetition suppression may play a role in the registration of more complex regularities as well. Indeed, at the neural level, a reduction of sensory responses in found for both repeated and predicted stimuli. Allenmark et al. (2015) inquire on the relationship between effects of stimulus repetition and effects of stimulus prediction, arguing that both should have similar behavioral consequences if they reflect the same underlying mechanism. However, at the behavioral level, the studies conducted so far have yielded enhanced performance for repeated stimuli, which afford faster and more accurate responses (a phenomenon known as repetition priming, see Schacter and Slotnick, 2004); while predicting a stimulus has been shown to impair performance (Waszak et al., 2012). Yet, perceptual sensitivity measures have been typically used in the prediction studies showing impaired performance, while repetition priming is typically measured in reaction time differences. Hence, Allenmark et al. (2015) measured intensity discrimination sensitivities for repeated compared to non-repeated stimuli. Moreover, they investigated the role of expectation on the repetition effects, by manipulating the probability of stimulus repetition. The results confirm that intensity discrimination sensitivity is also reduced for repeated stimuli, as it is for predicted stimuli. Additionally, they show that this stimulus repetition effect is influenced by predictive processes, as it is enhanced for expected compared to unexpected repetitions. These findings suggest that indeed prediction effects, in particular the sensory attenuation described for predicted stimuli, and the neural phenomenon of repetition suppression may be functionally related.

Sensory attenuation for predicted stimuli has been particularly described in the context of action-related prediction, explained as the cancellation of reafferent information making use of internally generated predictions about the expected sensory consequences of motor commands (reafference principle, see above). Following this interpretation, sensory suppression indices are often directly used as a measure to evaluate predictive processing capabilities, e.g. in clinical populations (e.g. Damaso et al., 2015). Horváth (2015) takes a reflective step back from this standpoint, questioning the assumption that sensory suppression effects for self-generated stimulation are due to predictive processes. He presents a lucid, critical review of self-generation studies, and convincingly argues that the evidence for this functional interpretation is limited. One important piece of evidence challenging the current consensus on motor-sensory suppression is the finding that contingent relations between actions and sensory effects are not necessary for a suppression effect to occur (Horvath et al., 2012). The review by Horváth should be a reminder to avoid the indiscriminate use of prediction explanations, without careful questioning of the alternatives.

Despite the fact that it may be possible to account for some of the self-generation sensory suppression effects without recurrence to predictive processes, other actionrelated phenomena are hard to explain without prediction (Waszak et al., 2012; Schröger et al., 2015). For example, it would be difficult to explain the accuracy that is displayed in tasks involving sensorimotor synchronization, e.g., catching a flying ball. Van der Steen et al. (2015) demonstrate the involvement of predictive processes in sensorimotor synchronization in a finger tapping task. They investigated how people synchronize their finger tapping to sound sequences that include tempo changes. This issue is relevant for musicians or dancers that need to coordinate their movement with each other. To perform such a synchronized tapping task, it is assumed that two different processes engage: adaptation and anticipation. The former is a reactive process of correcting errors in a post-hoc manner, whilst the latter is based on making predictions about when a sound will occur in time. Van der Steen et al. propose a model, in which these two mechanisms (adaptation and anticipationADAM model) are combined and can act synergistically through a joint module that links them. By comparing the behavioral results from the finger tapping tasks to the simulations obtained by using different versions of the ADAM, they tested how adaptation and anticipations mechanisms contribute to successful sensorimotor synchronization. Their main hypothesis, confirmed by their simulations, is that both these processes contribute to it, but more importantly, that the joint module plays an important role. Of note, in their study, predictive mechanisms seemed to be more engaged when tempo changes were larger, while they did not seem to play a big role in relatively small (in the order of 1-14 ms) tempo changes, where adaptation mechanisms seemed to play a more significant role.

Another example of action-related phenomena which can hardly be explained without prediction is the finding that unexpected omissions of self-generated stimuli cause brain activity. Activity elicited by omissions of strongly expected stimuli has been found to mirror the activity that is elicited by a stimulus when it is actually presented (Bendixen et al., 2009; SanMiguel et al., 2013a, b). These omission findings seem to unequivocally point to predictive processes, particularly supporting the idea that motor acts that afford predictions preactivate an image of their predicted sensory consequences (preactivation hypothesis, Waszak et al., 2012). This is also in accordance with predictive coding theories, in which the prediction error computed at a particular processing level corresponds to the difference between the sensory input into this level sent from the lower level and the prediction sent from the higher level. When there is no sensory input and a strong prediction, the prediction error is the same as when there is no prediction but only sensory input. For this reason, omission paradigms can be regarded 
as a kind of gold standard to unmask the neural representation of a prediction (for reviews see, Arnal and Giraud, 2012; Schröger et al., 2015). In this issue, Stekelenburg and Vroomen (2015) extended and compared the omission findings from the motor-sensory domain to the cross-modal visual-auditory domain, finding that there is an overlap between auditory predictions induced by motor- and visualauditory associations. That is, the early neural activity elicited by omissions of a sound was similar in amplitude, topography, and neural sources, irrespective of whether the omitted sound could be predicted on the basis of a button press or the image of a hammer hitting.

As we have argued throughout, prediction is in service of perception and action. The preactivation of sensory responses so elegantly illustrated in omission paradigms can be useful, for example, to enable a stable perceptual experience in the face of distortions and interruptions of the sensory signals, which are so common in the overcrowded and noisy sensory contexts of natural situations. In the auditory modality, for example, a common problem that perception has to solve is the issue of overlapping sound sources, such as the interruption of a sound object (e.g. the music we are listening to) that is temporarily masked by a louder sound (e.g. the noise of a truck passing by). It has been demonstrated that we may experience a continuity illusion when a segment of a sound sequence is replaced by noise, even when the sound sequence does not actually continue under the noise (Warren and Obusek, 1972). Bendixen et al. (2015) investigate the implication of predictive processes in the continuity illusion applying the logic of the omission studies. The hypothesis here is that whenever possible, sensory input is processed in a predictive manner, preactivating the upcoming input, and that the illusion of continuity would be enabled by this preactivation, which would substitute the actual sensory responses during brief interruptions of the input signal. Accordingly, ERPs elicited by noise stimuli that mask predictable tones should be more similar to ERPs elicited by the actual tones than when the same comparison is made for unpredictable tones. Although the results were partly in agreement with the prediction hypothesis, some methodological issues remain that need to be addressed before this innovative approach can be applied to further study the contribution of prediction in the processing of degraded sensory signals.

So far, we mostly have considered studies revealing that the human information processing system can exploit regularities in short-term history (temporary local context) to set up predictions. That is, in most studies described above, the regularities being tested are established from the immediately preceding input. However, studies of predictive processing in e.g. language (Huettig, 2015; Hisagi et al., 2015) or music (Guo and Koelsch, 2015) reveal that predictive models also include long-term implicit knowledge. In this issue, Sulykos et al. (2015) provide evidence for this exploring the effects of stimulus familiarity (i.e. long-term history) in a visual MMN study. They related their study to the phenomenon of search asymmetry, whereby certain visual objects are easier to detect among familiar than unfamiliar distractors (e.g. easier to detect a pseudo-letter among letters than vise versa). They reported a reduced vMMN latency for the unfamiliar deviant (a mirrored $\mathrm{N}$ ) presented in familiar standards (letter N) than vise versa. They argued that this is due to a more efficient encoding and access to the memory representation of familiar events. Thus, the familiarity effects would be based on the predictive model, being easier to construct and generate predictions via a familiar item. This is compelling electrophysiological evidence that the predictive processes contributing to visual perception do not only rely on the immediate sensory history (the stimulus context) but that they also consider regularities acquired through long-term training. In other words, the context information and long-term memory contents contribute to the same predictive model.

The contribution of long-term knowledge to predictive models should allow for more elaborated rules to play a role in the automatic generation of predictions, and hence for prediction to be involved in more complex functions. For example, syntactic rules may be used to generate predictions in the processing of language (see Hisagi et al., 2015, discussed in Section 4) or music (see Guo and Koelsch, 2015, discussed in Section 4). In this issue, Huettig (2015) examines the possibility that language processing is based on prediction mechanisms. This is a newly emerging approach (cf. Van Petten, 2012; Tavano and Scharinger, 2015; Winkler and Schröger, 2015), which counters the view of many linguists that prediction is not necessary as language users can rely on multiple options to derive meaning when encountering a word. In light of multiple psycholinguistic results to the contrary of the latter view, Huettig proposed that at least four mechanisms need to be considered when talking about prediction in language research: why prediction, what type of prediction, how does prediction work also in interaction with other cognitive processes such as working memory, and when in language processing input can be predicted. In a comprehensive and critical overview, Huettig presented conceptual development paired with empirical evidence on all four mechanisms subsumed under the term PACS (production, association, combinatorial and simulation-based prediction). In addition, he considered variables such as individual differences, age, working memory capacity etc. as important mediators in a prediction account of language processing.

In sum, we have seen how prediction is an innate capability of the human brain, which is ubiquitous in sensory processing across hierarchical levels and modalities, and is also involved in motor processes. The operation of internal models modulates stimulus-evoked responses, and has observable consequences in behavior. The specific neural mechanisms that underlie the formation and operation of internal models are as of yet unclear, but perhaps some clues can be found in the known neural adaptation mechanisms that take place in stimulus repetitions, and most likely a lot of what we already know regarding associative and other learning mechanisms could be incorporated to understand the formation of internal predictive models. The findings indicate that both local regularities and long term knowledge play a role in the generation of predictions, which can consequently be formulated at multiple (structural and functional) levels. The internal models also consider unimodal, 
crossmodal and motor-sensory regularities. Nevertheless, the resulting neural consequence of predicting a particular stimulus appears to be the same irrespective of the nature of the knowledge that led to the prediction, and it seems to consist on the activation of the neural code for the predicted stimulus.

\section{Attention}

Attention enables to select relevant rather than irrelevant information. Relevance is defined by the current goals of an organism, which can be set intentionally (e.g. via instruction) and involuntarily (e.g. via the context or hidden motives). In other words, attention exerts a topdown effect on information processing. The attentionfocused papers in this special issue considered different flavors of such top-down influence: voluntary and involuntary attention (Alho et al.; Hisagi et al.,), the impact of voluntary attention on early stages of information processing (Varghese et al.), the availability of processing resources (Watson et al.), familiarity or level of experience (musicians and non-musicians, Guo and Koelsch; native speakers vs. non-native speakers, Hisagi et al.; letters vs. pseudoletters, Sulykos et al.), higher-level expectations (often manipulated by explicit cuing or through manipulation of the relative probabilities of certain events or contingencies to take place-Parmentier and Kefauver; Allenmark et al.).

Alho et al. (2015) identified the neural networks involved in top-down controlled and in bottom-up triggered attention. Both types of attention activated overlapping brain areas including the superior temporal gyrus and sulcus, temporoparietal junction, superior parietal lobule, inferior and middle frontal gyri, frontal eye field, supplementary motor area, and anterior cingulate gyrus. According to recent definitions, bottom-up (involuntarily) triggered attention can be seen as generated by predictive processes, more specifically, involuntary attention comes into play when a prediction is violated by the incoming sound. Thus, this study suggests a partly overlapping network for attention and prediction. Moreover, they showed that fronto-parietal networks of attention to location or pitch overlap to a large extent. However, the anterior superior temporal cortex might have a more important role in attention to the pitch than to the location of sounds. As involuntary attention can best be explained in the prediction framework, the study of Alho et al. taps in the interaction of prediction and attention (which will be discussed in Section 4).

As introduced above, predictions can shape sensory processing at a relatively early stage of hierarchical processing (e.g. Cornella et al., 2015). Also attentional effects have been reported to occur along the auditory pathway, primary sensory cortices, subcortically (e.g. thalamus), and even at the cochlear level (for reviews see, e.g., Giard et al., 2000; Fritz et al., 2007). In their contribution, Varghese et al. (2015), investigated whether the envelope-following response (EFR), which is a particular kind of the subcortical steady state responses (SSRs), is affected by attention. The authors modulated attention in several ways, within the auditory modality in a dichotic listening task and in a crossmodal fashion by comparing EFRs when visual and auditory stimuli were attended. Similar to previous studies on the auditory brainstem responses (ABRs), they obtained a "null-effect" of the attentional manipulation in the ANOVAS for the EFRs, which was supported by a Bayes Factor analysis. This is interesting as an effect on the EFR could have been expected as attentional modulation of subcortically generated brain responses have been reported previously (see, e.g. Giard et al., 2000). Thus, they EFRs (and ABRs) seem to be rather robust against attentional manipulations.

In general, temporarily regular stimulation can induce neural entrainment (Henry et al., 2014) and can be used to generate appropriate expectations about the occurrence of a sound (e.g. Jones et al., 2010), which, in turn, may lead to improved performance (Rohenkohl et al., 2012). Thus, there seems to be a close link between temporal regularity in stimulus presentation, oscillatory brain activity, and attentional/perceptual effects, which are examined in three contributions to this special issue. In a seminal review, Gregoriou et al. (2015) discuss findings that link neural synchrony with attention with a focus on non-human primates and the visual system. The authors explained the role of neural synchrony as a mechanism to enhance processing of selected inputs and as a means to achieve effective communication between distant neural populations representing behaviorally relevant stimuli. They reviewed the existing evidence by showing that changes in neural synchrony correlate with attentional benefits observed in behavior. The authors described cellular and circuit mechanisms that give rise to network oscillations and reviewed findings on how attention affects network oscillations. The evidence shows that attention modulates oscillatory synchrony both locally and between distant populations. The authors also evaluated findings on the influence of neurotransmitters in attention, concluding that acetylcholine is particularly involved in the modulation of oscillatory activity, contributing to attention effects. In a complementary review, Frey et al. (2015) present evidence that oscillatory brain activity in humans is modulated by attention. By jointly considering various theories relating oscillations to attention and the results of a number of attention paradigms involving spatial, temporal, selective, and internal attention they identified two oscillatory processes in attention: one that is expressed in power modulations (preferentially in the alpha band) and seems to reflect a kind of continuous attention mode, while the other is expressed in phase modulations (e.g. entrainment) in lower frequency bands (delta and beta bands) and seems to reflect a rhythmic processing mode. They integrate their findings with results from human research and the model proposed by Schroeder and Lakatos (2009) based on animal research. Finally, Bauer et al. (2015) tested whether variations of a particular pitch comparison task are suited to investigate the relation between rhythmic auditory stimulation and task accuracy. This would be a most preferable approach as the sound dimension for which the behavioral performance is determined, is independent of the temporal dimension resulting in the fluctuations of attention. Five carefully conducted experiments 
yielded null-effects suggesting that the pitch comparison task is not optimally suited for demonstrating auditory dynamic attending.

In sum, attention networks overlap with prediction networks; similar to prediction, effects of attention can occur quite early in terms of time and structure, and not only prediction but also attention modulates oscillatory activity in the brain. Thus it is more than logical, to have a closer look at the interaction between prediction and attention, which we will do in the next chapter.

\section{Interactions between attention and prediction}

Prediction and attention both enable or aid perception and action. While some of the contributions in this special issue shortly described above, were devoted to either prediction or attention, others already revealed a close link between the two concepts. In fact, several contributions to this special issue explicitly addressed a potential interaction of prediction and attention, which has not been sufficiently addressed so far. In principle, many attentional phenomena can be integrated into a predictive coding framework (e.g. Schröger et al., 2015). Predictive coding theories (e.g. Feldman and Friston, 2010) postulate a predictive model that generates (feedback) inferences on the content of and the confidence in the sensory evidence. The difference between input and prediction is expressed in a prediction error, which is a (feedforward) signal that updates the model and, thus, helps to reduce the ambiguity with respect to how we interpret the world. Attention affects the precision of the prediction for the attended content via the model by modulating the gain of the prediction error. This improves the model and-as a consequence-solves the perceptual problem. If we consider perception as an active inference process, and accept that attention modulates the neural signals involved in this predictive process, it is clear that attention and prediction should interact. This interaction can take two forms: (i) attention affecting predictive processes and (ii) predictive processes driving attention.

The question of attentional modulation of predictive processes is intermingled with a long standing debate regarding the distinction between controlled and automatic processes. Most of the predictive processes that we have discussed so far are regarded as being mostly automatic, in the sense that they are an integral part of lower-level sensory processing and do not require conscious or effortful control to take place. However, some studies in this special issue are also a testimony of the fact that prediction is not only an issue of lower level sensory processing but also of higher level cognitive processing, where prediction may be more or less dependent on controlled processes. Moreover, even for the most automatically occurring, lowest-level predictions (e.g. MLR responses in Cornella et al., 2015) it is still a valid question to ask whether top-down factors, which can be conceived as attentional, can modulate predictive processes. Top-down factors might impact both processes whereby regularities are discovered, and the effects that the internal models of those regularities have on subsequent sensory processing. The contribution of Watson et al. (2015) addresses the first of these two possibilities, investigating the formation of action-related associations. After establishing an actionoutcome association, presenting the associated outcome facilitates the execution of an action, while there are costs for performing incongruent actions. Watson et al. (2015) asked the question whether the formation of these associations is fully automatic, or whether it depends on the availability of limited resources (i.e. attention, see Section 3). This is related to the important issue of whether the acquisition of action-outcome associations happens incidentally, or whether it requires the intention to learn. Their findings suggest that associations can be acquired incidentally only in relatively simple settings, where few actionoutcome pairings are available simultaneously. This denotes that the extraction of this type of regularity (action-outcome pairings) requires attentional resources, thus differing from findings in the auditory regularity extraction literature. MMN studies have shown that auditory regularities are extracted largely automatically and can even be found in newborn infants (see e.g. Háden et al., 2015) or in participants being strongly engaged in another task (for a critical review, see, e.g., Sussman et al., 2014). Nevertheless, it is still possible that action-outcome associations can be encoded automatically, but attention is required for these associations to have a lasting impact on subsequent behavior, i.e, to be able to measure congruence effects in behavior.

While attention is not required to elicit a MMN, the MMN amplitude can be modulated by attention. Three contributions in this special issue (Hisagi et al., Sulykos et al., Guo and Koelsch) investigated the modulation by top-down factors of electrophysiological signals elicited by the violation of regularities. Hisagi et al. (2015) investigated the interaction of attention, prediction, and familiarity by assessing the influence of attention on deviance detection as a function of the level of familiarity or prolonged experience with a stimulus. The authors used MMN to examine the influence of attention on the processing of phonetic contrasts in native and nonnative speakers in order to test whether speech perception in a native language is highly automatic. Their results revealed that when attention was directed to the stimuli, there was an increased $\mathrm{MMN}$ in both native and non-native Japanese listeners in a consonant length contrast. They concluded that the level of automaticity might depend on the difficulty of the specific contrast (consonants vs. vowels), and even for native speakers attention may play a role in processing perceptually more difficult contrasts. These results are also in agreement with another form of top-down influence on deviance processing, namely familiarity (see Sulykos et al., 2015, discussed in Section 2).

Guo and Koelsch (2015) took a closer look at the influence of controlled processes, which form higher level expectancies or explicit knowledge, on lower level, automatic processes (predictions) in music. This approach is akin to previous studies that have investigated the influence of higher level expectancies on MMN (e.g. based on cues or manipulations of the probability of deviance), but applied to specific processes in music. However, one important difference needs to be noted: while the MMN reflects a change in regularities that are learned "on the fly" over the course of an experiment, the 
component of interest, ERAN, is elicited in response to unexpected changes of implicit, long-term knowledge of syntactic regularities in music. Even so, this information can be acquired automatically, is observable in non-musicians as well, and the rules are remarkably stable. The authors used violations of syntactic expectancies in music and attempted to modulate the responses elicited by the violations of (almost hard-wired) expectancies via repetition and explicit instruction that violations will occur. They tested musicians and non-musicians and found that the P300/LPC and P3a was modulated by learning, but not the ERAN. They conclude that acquiring higher-level expectancies does not influence the fast and partly automatic processing of implicit knowledge of musical regularities (ERAN) but that learning affects later controlled processes. Of note is that effects of learning as evident in the late ERPs (P3a/LPC) were observable even in non-musicians who did not show behavioral signs of learning.

So far we have discussed evidence on the modulation of predictive processes by top-down factors related to attention. We now turn to interactions between attention and prediction that go in the opposite direction, that is, the impact of predictive processes on attention. One of the major challenges for cognitive psychology and neuroscience research is to be able to explain processes, which are placed at the top of the control hierarchy without recurrence to a higher-order decision making system. In relation to this, an outstanding question in the field of attention is to explain how the direction of attention is controlled. Predictive processes may be a major force in driving the direction of attention. In the predictive coding view, attention is directly linked to prediction, as gain modulations are tied to confidence in the sensory evidence, i.e., to an estimation of how accurate the predictions stemming from the internal model can be at a given moment. Albeit somewhat indirectly, prediction has long been implicated in driving attention in classic experimental psychology research. Traditionally, probabilistic cues have been used to direct attention in Posner-type cuing paradigms. In these studies, it is implicitly assumed that attention is directed towards stimuli, which are predicted with a certain level of accuracy by the cues. However, perhaps how prediction drives attention is even more clear in involuntary attention studies, where unexpected stimuli are employed to elicit an orienting response, i.e., the involuntary driving of attention. For example, Schröger (1996) paired a classic oddball paradigm with a reaction time task, demonstrating that deviant stimuli not only elicit the well-known deviance detection signals such as the MMN, but also cause momentary distraction (RT and error rate increases) by involuntarily capturing attention. Parmentier et al. (2011) later showed that deviant stimuli distract specifically because they violate predictions and not because they are rare per se, and, in fact, deviance distraction is reduced when deviant sounds are predicable. In the current issue, Parmentier and Kefauver address different ways, in which a prediction violation can cause distraction, by investigating the involvement of predictive processes in a semantic effect. Deviant sounds cause a delay in the processing of target stimuli by momentarily capturing attention away from the task-relevant information (Parmentier et al., 2008). The involuntary attention shift towards the deviant stimulus also forces the involuntary semantic processing of the deviant, thus, semantic interference can occur if the deviant stimulus is incongruent with the task-relevant stimulation (e.g. the deviant distractor is the word "left", but the following target requires a "right" response). In their contribution, Parmentier and Kefauver (2015) showed that semantic interference decreased as the proportion of semantically incongruent trials increased, concluding that prediction also plays a major role in semantic interference effects. This is interesting, because related interference effects like e.g. the Stroop effect, or the Simon effect, have not typically been interpreted from the prism of prediction. Nevertheless, Parmentier and Kefauver's findings highlight the fact that in their study, predictions seemed to be automatically formulated not only regarding the nature of the upcoming stimuli, but also as response anticipations. That is, the semantic effect likely reflects the interference caused by anticipating a response which upon target presentation turned out to be the wrong one.

However, such evidence for stimulus or response anticipations is not necessarily obtained in all circumstances. Although animal research revealed that receptive fields of neurons are remapped prior to and during saccadic eye movements to match the new locations of visual stimuli during the next fixation (Nakamura and Colby, 2002), this seem not to apply for humans. Grubert and Eimer (2015) tested whether human visual working memory represents the predicted locations of future target objects. If it would, the contralateral delay activity (CDA) of the ERP should be elicited in the critical experimental conditions. However, the finding that CDA components are elicited contralateral to the side where task-relevant objects appeared in a memory display (and not to the side of the upcoming comparison stimuli) suggests that object representations in visual working memory are position-dependent representing the location during encoding and that they cannot be flexibly shifted towards predicted locations of future target events.

Understanding the interaction between attention and prediction can have a substantial impact on clinical research. Attention symptoms are commonplace in many neurological and psychiatric disorders, and their specific etiology is rarely understood. Dysfunctions of the prediction mechanisms, or disturbances in the appropriate interplay between attentive and predictive processes may therefore explain many attention symptoms. For example, attentional deficits are a core feature of schizophrenia. In previous research, patients suffering from schizophrenia were reported to differ from healthy controls in their ability to properly form sensory predictions (e.g. Ford and Mathalon, 2012), and in MMN measures elicited by events violating regularity (Todd et al., 2012). In this special issue, Damaso et al. (2015) reviewed findings on the modulation of MMN in persons with schizophrenia and attempted to relate these findings to observed attention deficits. The authors proposed that in schizophrenia there might be a failure in the confidence-based modulation of the error signal (the gain control), that is, a failure to appropriately accumulate confidence in the predictive model, leading to a reduced upper limit of MMN amplitude. They then considered how this alteration in the MMN-eliciting predictive system might affect attentional control. It is known that larger MMNs are associated with stronger orienting responses and the disruption of task performance. 
However, the relationship between MMN amplitude and attention is not straightforward. Damaso et al. proposed that a failure of the gain control of the error signal could mean that persons with schizophrenia are less able to determine sound relevance, ultimately leading to greater attentional disruptions.

\section{Conclusions}

The goal of this special issue was to discuss the sometimes diverse and contradictory concepts of prediction and attention in perception and action. For example, what has been called attention in older attentional cuing studies is nowadays often called prediction, and attention is more confined to settings where particular stimulus features are defined as task relevant, that is, attended to (in contrast to task-irrelevant, i.e. unattended, stimulus features). Moreover, the large field of involuntary attention, where attention is captured by irregular events, is more and more explored under the heading of prediction. As one can see from the multitude of contributions, the topic remains complex. The different contributions show how closely linked the concepts of attention and prediction are, but also how diverse and still unresolved some of the main questions under the respective headings are. They also reveal how these concepts have been applied to many different domains of perception and action. Despite the fact that in some contexts their definitions overlap and that prediction and attention closely link in how they relate to perception and action, we hope to provide rather cohesive evidence that attention and prediction should be considered as differentiated processes. Further, many of the contributions in this special issue explore the interaction between attention and prediction, a newly emerging and highly challenging topic.

Altogether, there seems to be broad consensus on some issues. First, there is sufficient evidence that the brain operates in a predictive mode to optimize perception and action, which is-in essence-nothing more than exploiting what we already know about the world to manage new situations and events. That is, prediction is a general working principle of the brain. Second, given the overflow of information our cognitive systems have to deal with, there seems to be no discussion on the necessity of a general brain function that can prioritize certain aspects of information. This is the core concept of attention. Because information is, by default processed in a predictive manner, in our view, attention can prioritize information by modulating predictive processing. In doing so, attention can interface at different levels along the processing hierarchy and also in various ways. We have mainly considered (voluntary and involuntary) selective attention, but other forms of attention such as the inherent dynamics of the attentional system, vigilance and arousal, and the diversity of executive functions also deserve to be taken into account.

However, a lot of issues remain open. Importantly, despite the agreement that attention and prediction exist, operational and consensual definitions of these terms are needed for the advancement of the field. These definitions may be based on conceptual, cognitive constructs, so long as they are clearly defined. One proposal along these lines would be that of Summerfield and Egner (2009), see also Schröger et al.
(2015), to define prediction in terms of probability and attention in terms of relevance. Another option, however, could be to deduct definitions based on their neural effects. This alternative has been adopted under the predictive coding framework (Feldman and Friston, 2010), where prediction is, per definition, the feedback from higher (more central) to lower (more peripheral) areas, while the term attention is reserved for gain modulations of the feedforward signal. Independently of how we define the terms, another important question that remains open is what determines where and when gain should be enhanced (according to the second definition of attention), or what determines that a particular piece of information is relevant (according to the first definition). That is, we still do not fully understand what drives attention, which cannot be attention itself.

A promising venue that might help provide answers to many of these long-standing issues, is the study of oscillations. More and more, researchers consider a relation between oscillatory processes in the brain and attention (e.g., Gregoriou et al. and Frey et al., 2015). As a periodic signal that can become synchronized with external signals, oscillations are also an excellent means to be employed in predictive processing. And indeed, particularly when dealing with predictions regarding the timing of events, oscillatory mechanisms have received a prominent role. An important issue in this respect, which is rarely addressed, might be the differentiation of "what" and "when" in attention and prediction. That is, to understand, on the one hand, the functional mechanisms that enable temporal predictions, and the focusing of attention at particular time points; and on the other hand, the functional mechanisms that allow to focus attention on, and to predict, particular objects or features (Schwartze et al., 2012; Schwartze and Kotz, 2013).

All in all, many questions remain regarding the neural mechanisms that underlie the constructs of attention and prediction in the brain. In the field of prediction, one very important open question refers to how internal predictive models are formed. For example, it is still unclear what the role of neural adaptation is in prediction, or how rules are encoded at all. In this respect, it would be rather interesting to try to interface these issues with the accumulated knowledge that we already have in the fields of learning, the neural basis of associations, and priming.

We hope that bringing together work from different traditions, which have dealt with diverse definitions of the concepts of prediction and attention, we help to promote a more integrative understanding of these complex issues and can encourage researchers interested in these constructs to keep an open mind in understanding alternative views. In particular, it should be very fruitful to closely examine the links and similarities between the effects and the concepts being used in different traditions under different headings, and make attempts to speak a common language, so that the knowledge can be integrated. We aimed at establishing some common ground and apparent connections between concepts here. Finally, we wanted to highlight possible interactions between attention and prediction. This is a promising emerging field, which we believe may hold the key to understand many of the long standing queries, such as what 
controls the direction of attention or what underlies commonplace attentional deficits in various clinical populations.

\section{Acknowledgments}

This work was funded by a Reinhart-Koselleck grant of the German Research Foundation SCHR 375/20 (Deutsche Forschungsgemeinschaft, DFG) to ES and by the Spanish Ministry of Economy and Competitiveness (MINECO) through Ramón y Cajal fellowship RYC-2013-12577 to ISM.

\section{R E F E R E N C E S}

Adams, R.A., Shipp, S., Friston, K.J., 2013. Predictions not commands: active inference in the motor system. Brain Struct. Funct. 218 (3), 611-643.

Alho, K., Salmi, J., Koistinen, S., Salonen, O., Rinne, T., 2015. Topdown controlled and bottom-up triggered orienting of auditory attention to pitch activate overlapping brain networks. Brain Res. 1626, 136-145.

Allenmark, F., Hsu, Y.-F., Roussel, C., Waszak, F., 2015. Repetition priming results in sensitivity attenuation. Brain Res. 1626, 211-217.

Arnal, L.H., Giraud, A.L., 2012. Cortical oscillations and sensory predictions. Trends Cogn. Sci. 16 (7), 390-398.

Bauer, A.-K.R., Jaeger, M., Thorne, J.D., Bendixen, A., Debener, S., 2015. The auditory dynamic attending theory revisited: a closer look at the pitch comparison task. Brain Res. 1626, 198-210.

Bendixen, A., SanMiguel, I., Schröger, E., 2012. Early electrophysiological indicators for predictive processing in audition: aA review. Int. J. Psychophysiol. 83 (2), 120-131.

Bendixen, A., Duwe, S., Reiche, M., 2015. Noise occlusion in discrete tone sequences as a tool towards auditory predictive processing?. Brain Res. 1626, 97-107.

Bendixen, A., Schröger, E., Winkler, I., 2009. I heard that coming: event-related potential evidence for stimulus-driven prediction in the auditory system. J. Neurosci. 29 (26), 8447-8451.

Clark, A., 2013. Whatever next? Predictive brains, situated agents, and the future of cognitive science. Behav. Brain Sci. 36 (3), 181-204.

Cornella, M., Bendixen, A., Grimm, S., Leung, S., Schröger, E., Escera, C., 2015. Spatial auditory regularity encoding and prediction: Human middle-latency and long-latency auditory evoked potentials. Brain Res. 1626, 21-30.

Damaso, K.A.M., Michie, P.T., Todd, J., 2015. Paying attention to MMN in schizophrenia. Brain Res. 1626, 267-279.

Escera, C., Alho, K., Schröger, E., Winkler, I., 2000. Involuntary attention and distractibility as evaluated with event-related brain potentials. Audiol. Neuro-Otol. 5 (3-4), 151-166.

Feldman, H., Friston, K.J., 2010. Attention, uncertainty, and freeenergy. Front. Human Neurosci., 4.

Ford, J.M., Mathalon, D.H., 2012. Anticipating the future: automatic prediction failures in schizophrenia. Int. J. Psychophysiol. 83 (2), 232-239.

Friedman, D., Cycowicz, Y.M., Gaeta, H., 2001. The novelty P3: an event-related brain potential (ERP) sign of the brain's evaluation of novelty. Neurosci. Biobehav. Rev. 25 (4), 355-373.

Frey, J.N., Ruhnau, P., Weisz, N., 2015. Not so different after all: The same oscillatory processes support different types of attention. Brain Res. 1626, 183-197.

Friston, K.J., 2005. A theory of cortical responses. Philos. Trans. R. Soc. B-Biol. Sci. 360 (1456), 815-836.
Friston, K.J., Daunizeau, J., Kilner, J., Kiebel, S.J., 2010. Action and behavior: a free-energy formulation. Biol. Cybern. 102 (3), 227-260.

Fritz, J.B., Elhilali, M., David, S.V., Shamma, S.A., 2007. Auditory attention-focusing the searchlight on sound. Curr. Opin. Neurobiol. 17 (4), 437-455.

Giard, M.H., Fort, A., Mouchetant-Rostaing, Y., Pernier, J., 2000. Neurophysiological mechanisms of auditory selective attention in humans. Front. Biosci. 5, D84-D94.

Gregoriou, G.G., Paneri, S., Sapountzis, P., 2015. Oscillatory synchrony as a mechanism of attentional processing. Brain Res. 1626, 165-182.

Grill-Spector, K., Henson, R., Martin, A., 2006. Repetition and the brain: neural models of stimulus-specific effects. Trends Cogn. Sci. 10 (1), 14-23.

Grimm, S., Escera, C., 2012. Auditory deviance detection revisited: evidence for a hierarchical novelty system. Int. J. Psychophysiol. 85 (1), 88-92.

Grubert, A., Eimer, M., 2015. Does visual working memory represent the predicted locations of future target objects? An event-related brain potential study. Brain Res. 1626, 258-266.

Guo, S., Koelsch, S., 2015. The effects of supervised learning on event-related potential correlates of music-syntactic processing. Brain Res. 1626, 232-246.

Háden, G.P., Németh, R., Török, M., Winkler, I., 2015. Predictive processing of pitch trends in newborn infants. Brain Res. 1626, 14-20.

Haenschel, C., Vernon, D.J., Dwivedi, P., Gruzelier, J.H., Baldeweg, T., 2005. Event-related brain potential correlates of human auditory sensory memory-trace formation. J. Neurosci. 25 (45), 10494-10501.

Helmholtz, H. v, 1867. Handbuch der physiologischen Optik. Voss, Leipzig.

Henry, M.J., Herrmann, B., Obleser, J., 2014. Entrained neural oscillations in multiple frequency bands comodulate behavior. Proc. Natl. Acad. Sci. USA 111 (41), 14935-14940.

Hisagi, M., Shafer, V.L., Strange, W., Sussman, E.S., 2015. Neural measures of a Japanese consonant length discrimination by Japanese and American English listeners: Effects of attention. Brain Res. 1626, 218-231.

Hohwy, J., 2013. The Predictive Mind. University Press, Oxford.

Horváth, J., 2015. Action-related auditory ERP attenuation: paradigms and hypotheses. Brain Res. 1626, 54-65.

Horvath, J., Maess, B., Baess, P., Toth, A., 2012. Action-sound coincidences suppress evoked responses of the human auditory cortex in EEG and MEG. J. Cogn. Neurosci. 24 (9), 1919-1931.

Huettig, F., 2015. Four central questions about prediction in language processing. Brain Res. 1626, 118-135.

Hughes, G., Desantis, A., Waszak, F., 2013. Mechanisms of intentional binding and sensory attenuation: the role of temporal prediction, temporal control, identity prediction, and motor prediction. Psychol. Bull. 139 (1), 133-151.

James, W., 1890. Principles of Psychology. Holt, New York, NY. Jones, S.R., Kerr, C.E., Wan, Q.A., Pritchett, D.L., Hamalainen, M., Moore, C.I., 2010. Cued spatial attention drives functionally relevant modulation of the mu rhythm in primary somatosensory cortex. J. Neurosci. 30 (41), 13760-13765.

Kimura, M., Takeda, Y., 2015. Automatic prediction regarding the next state of a visual object: Electrophysiological indicators of prediction match and mismatch. Brain Res. 1626, 31-44.

Kok, P., Rahnev, D., Jehee, J.F.M., Lau, H.C., de Lange, F.P., 2012. Attention reverses the effect of prediction in silencing sensory signals. Cereb. Cortex 22 (9), 2197-2206.

Kujala, T., Tervaniemi, M., Schröger, E., 2007. The mismatch negativity in cognitive and clinical neuroscience: theoretical and methodological considerations. Biol. Psychol. 74 (1), 1-19. 
Lee, T.S., Mumford, D., 2003. Hierarchical Bayesian inference in the visual cortex. J. Opt. Soc. Am. a-Opt. Image Sci. Vis. 20 (7), 1434-1448.

May, P.J.C., Tiitinen, H., 2010. Mismatch negativity (MMN), the deviance-elicited auditory deflection, explained. Psychophysiology 47 (1), 66-122.

Miall, R.C., Wolpert, D.M., 1996. Forward models for physiological motor control. Neural Netw. 9 (8), 1265-1279.

Näätänen, R., 1990. The role of attention in auditory informationprocessing as revealed by event-related potentials and other brain measures of cognitive function. Behav. Brain Sci. 13 (2), 201-232.

Näätänen, R., 1992. Attention and Brain Function. Erlbaum, Hillsdale, NJ.

Näätänen, R., Alho, K., Schröger, E., 2002. Electrophysiology of Attention. In: Pashler, H., Wixted, J. (Eds.), Steven's Handbook of Experimental Psychology, Third Edition Volume Four: Methodology in Experimental Psychology. John Wiley, New York, pp. 601-653.

Näätänen, R., Kujala, T., Winkler, I., 2011. Auditory processing that leads to conscious perception: a unique window to central auditory processing opened by the mismatch negativity and related responses. Psychophysiology 48 (1), 4-22.

Näätänen, R., Winkler, I., 1999. The concept of auditory stimulus representation in cognitive neuroscience. Psychol. Bull. 125 (6), 826-859.

Nakamura, K., Colby, C.L., 2002. Updating of the visual representation in monkey striate and extrastriate cortex during saccades. Proc. Natl. Acad. Sci. USA 99 (6), 4026-4031.

Parmentier, F.B.R., Kefauver, M., 2015. The semantic aftermath of distraction by deviant sounds: Crosstalk interference is mediated by the predictability of semantic congruency. Brain Res. 1626, 247-257.

Parmentier, F.B.R., Elford, G., Escera, C., Andrés, P., Miguel, I.S., 2008. The cognitive locus of distraction by acoustic novelty in the cross-modal oddball task. Cognition 106 (1), 408-432.

Parmentier, F.B.R., Elsley, J.V., Andres, P., Barcelo, F., 2011. Why are auditory novels distracting? Contrasting the roles of novelty, violation of expectation and stimulus change. Cognition 119 (3), 374-380.

Polich, J., 2007. Updating P300: an integrative theory of P3a and P3b. Clin. Neurophysiol. 118 (10), 2128-2148.

Rao, R.P.N., Ballard, D.H., 1999. Predictive coding in the visual cortex: a functional interpretation of some extra-classical receptive-field effects. Nat. Neurosci. 2 (1), 79-87.

Rohenkohl, G., Cravo, A.M., Wyart, V., Nobre, A.C., 2012. Temporal expectation improves the quality of sensory information. J. Neurosci. 32 (24), 8424-8428.

SanMiguel, I., Todd, J., Schröger, E., 2013a. Sensory suppression effects to self-initiated sounds reflect the attenuation of the unspecific N1 component of the auditory ERP.

Psychophysiology 50 (3), 334-343.

SanMiguel, I., Widmann, A., Bendixen, A., Trujillo-Barreto, N., Schröger, E., 2013b. Hearing silences: human auditory processing relies on pre-activation of sound-specific brain activity patterns. J. Neurosci. 33 (20), 8633-8639.

Schacter, D.L., Slotnick, S.D., 2004. The cognitive neuroscience of memory distortion. Neuron 44 (1), 149-160.

Schroeder, C.E., Lakatos, P., 2009. Low-frequency neuronal oscillations as instruments of sensory selection. Trends Neurosci. 32, 9-18.

Schröger, E., 1996. A neural mechanism for involuntary attention shifts to changes in auditory stimulation. J. Cogn. Neurosci. 8, 527-539.
Schröger, E., Marzecova, A., SanMiguel, I., 2015. Attention and prediction in human audition: a lesson from cognitive psychophysiology. Eur. J. Neurosci. 41 (5), 641-664.

Schwartze, M., Kotz, S.A., 2013. A dual-pathway neural architecture for specific temporal prediction. Neurosci. Biobehav. Rev. 37 (10 Pt 2), 2587-2596.

Schwartze, M., Tavano, A., Schröger, E., Kotz, S.A., 2012. Temporal aspects of prediction in audition: cortical and subcortical neural mechanisms. Int. J. Psychophysiol. 83 (2), 200-207.

Stekelenburg, J.J., Vroomen, J., 2015. Predictive coding of visualauditory and motor-auditory events: An electrophysiological study. Brain Res. 1626, 88-96.

Sulykos, I., Kecskés-Kovács, K., Czigler, I., 2015. Asymmetric effect of automatic deviant detection: The effect of familiarity in visual mismatch negativity. Brain Res. 1626, 108-117.

Summerfield, C., Egner, T., 2009. Expectation (and attention) in visual cognition. Trends Cogn. Sci. 13 (9), 403-409.

Summerfield, C., Trittschuh, E.H., Monti, J.M., Mesulam, M.M., Egner, T., 2008. Neural repetition suppression reflects fulfilled perceptual expectations. Nat. Neurosci. 11 (9), 1004-1006.

Sussman, E.S., Chen, S., Sussman-Fort, J., Dinces, E., 2014. The Five Myths of MMN: redefining How to Use MMN in Basic and Clinical Research. Brain Topogr. 27 (4), 553-564.

Tavano, A., Scharinger, M., 2015. Prediction in speech and language processing. Cortex 68, 1-7.

Todd, J., Michie, P.T., Schall, U., Ward, P.B., Catts, S.V., 2012. Mismatch negativity (MMN) reduction in schizophreniaImpaired prediction-error generation, estimation or salience?. Int. J. Psychophysiol. 83 (2), 222-231.

van der Steen, M.C., Jacoby, N., Fairhurst, M.T., Keller, P.E., 2015. Sensorimotor synchronization with tempo-changing auditory sequences: Modeling temporal adaptation and anticipation. Brain Res. 1626, 66-87.

Varghese, L., Bharadwaj, H.M., Shinn-Cunningham, B.G., 2015. Evidence against attentional state modulating scalp-recorded auditory brainstem steady-state responses. Brain Res. 1626, 146-164.

von Holst, E., Horst, M., 1950. Das reafferenzprinzip. Naturwissenschaften 37, 464-476.

Watson, P., van Steenbergen, H., de Wit, S., Wiers, R.W., Hommel, B., 2015. Limits of ideomotor action-outcome acquisition. Brain Res. 1626, 45-53.

Warren, R.M., Obusek, C.J., 1972. Auditory induction-illusionary continuity of fainter of 2 alternating sounds. J. Acoust. Soc. Am. 51 (1), 114.

Waszak, F., Cardoso-Leite, P., Hughes, G., 2012. Action effect anticipation: neurophysiological basis and functional consequences. Neurosci. Biobehav. Rev. 36 (2), 943-959.

Winkler, I., 2007. Interpreting the mismatch negativity. J. Psychophysiol. 21 (3-4), 147-163.

Winkler, I., Schröger, E., 2015. Auditory perceptual objects as generative models: setting the stage for communication by sound. Brain Lang. 148, 1-22.

Erich Schröger* Institute of Psychology, University of Leipzig, D-04103 Leipzig, Germany E-mail address: schroger@uni-leipzig.de

Sonja A. Kotz ${ }^{* *}$ Max Planck Institute for Human Cognitive and Brain Sciences, Department of Neuropsychology, P. O. Box 500 355, D-04303 Leipzig, Germany

*Corresponding author.

**Corresponding author at: Max Planck Institute for Human Cognitive and Brain Sciences, P.O. Box 500 355, D-04303 Leipzig, Germany 
Faculty of Psychology \& Neuroscience, Department of Neuropsychology \& Psychopharmacology, University of Maastricht, Maastricht, The Netherlands E-mail addresses: kotz@cbs.mpg.de, sonja.kotz@maastrichtuni versity.nl

Iria SanMiguel ${ }^{* * * * *}$ Institute for Brain Cognition and Behavior (IR3C) and Department of Psychiatry and Clinical Psychobiology, University of Barcelona, 08035 Barcelona, Spain E-mail address: isanmiguel@ub.edu 0006-8993/\$-see front matter (c) 2015 Elsevier B.V. All rights reserved.

http://dx.doi.org/10.1016/j.brainres.2015.08.037

**** Correspondence to: Brainlab - Cognitive Neuroscience Research Group, Department of Psychiatry and Clinical Psychobiology, University of Barcelona, P. Vall d'Hebron 171, 08035 Barcelona, Spain 